\title{
On De Broglie’s Double-particle Photon Hypothesis
}

\section{André Michaud*}

Senior Researcher, Canada

\begin{abstract}
Establishment of an LC equation and of a local fields equation describing permanently localized photons from the analysis of kinetic energy circulation within the energy structure of the double-particle photon that Louis de Broglie hypothesized in the early 1930's. Among other interesting features, these equations provide a mechanical explanation to the localized photon properties of self-propelling at the speed of light and of self-guiding in straight line when no external interaction tends to deflect its trajectory. This paper summarizes the seminal considerations that led to the development of the 3-spaces model.
\end{abstract}

Keywords: Electromagnetic theory; Kinetic energy; Photon; Acceleration; Electron-positron pairs; $1.022 \mathrm{MeV}$ LC equation; 3-spaces

\section{Introduction}

The first integrated representation of electromagnetic energy was provided by Maxwell as a continuous wave phenomenon that would be due to interacting electric and magnetic fields inducing each other, which led to the recognition that radio frequencies belong to the same electromagnetic spectrum as visible light. Then came Planck's analysis of Wien's experimental data on the black body demonstrating that electromagnetic energy is always captured as frequency dependent discrete amounts. Einstein's photoelectric proof confirmed Planck's hypothesis shortly afterwards by demonstrating that photons do behave as if they were separate localized quanta when intercepted while also demonstrating that they possess longitudinal inertia, which eventually earned them both Nobel prizes. Compton and Raman added further experimental confirmation of Planck's conclusion, while experimenting with other types of collisions between photons and electrons. These findings conclusively confirmed the discrete and point-like behavior of photons when being absorbed. We must also keep in mind that an interaction cross-section always larger than zero needs to be assumed for all point-like behaving particles during scattering experiments to correctly account for the observed recorded traces. So we know that photons are not really point-like with zero dimensions in the mathematical sense, even if their motion can be calculated as if they were; just like the trajectory of the Moon about the Earth is calculated as if their masses were concentrated in a single point at the center of each body. Point-like behavior of photons upon emission was also subsequently understood and verified, which we will have a look at further on. So, we know for certain that Maxwell's continuous "waves" do not exist as such at the submicroscopic level, despite the fact that his equations allow calculating all electromagnetic manifestations with the utmost precision when electromagnetic energy is treated as being continuous and featureless as observed from our macroscopic perspective.

In fact, what these discoveries reveal is that we are in the very same situation with respect to electromagnetic energy that we are in with respect to solid materials, as it closely parallels the fact that although we can observe that the surface of a polished diamond has a flawlessly smooth finish from our macroscopic perspective, for example, we can also alternately observe that this same surface is granular and bumpy when the scattering particles of an electron microscope reveal the outlines of the individual atoms making up the crystal surface at the submicroscopic level. In the latter case however, we have a rather extensive understanding of the inner structure of the atoms involved, but to this date, the inner structure of photons is still the object of speculation.

For the past century, there has been a deeply ingrained conception in the case of light that it sometimes behaves as a wave and sometimes as a particle, two types of behavior that are incompatible for a number of reasons and that gave rise to the concept of "wave-particle behavior" to characterize the photon. Close examination of the concept in light of the macroscopic-submicroscopic comparison just clarified leads to the view that generally speaking, "wave behavior" could simply be the result of behavior of crowds of discrete photons that our macroscopic instruments generally deal with while "particle behavior" could simply be the behavior of individual photons at the submicroscopic level. This would go a long way in removing the inherent incompatibility of the "wave-particle behavior" concept, by replacing it with a "macroscopicwave behavior vs submicroscopic particle behavior" concept. But we will see further on that with the model that will be proposed here, even at the submicroscopic level, the localized photon can display both types of behavior without any conflict by associating transverse wave behavior with longitudinal particle behavior. Also, despite its systematic pointlike behavior in all scattering and capture experiments, behavior typical of elementary particles, the photon was suspected early on of not being elementary because light can be polarized, which cannot be explained if the photon was made up of a single point-like behaving particle. This was clarified by Louis de Broglie as the concept of spin was introduced, associating a spin of $1 / 2$ to point-like behaving particles that were proven out of any doubt to be really elementary, such as the electron and the positron, and consequently a spin of 1 to the photon, thus hypothesizing that if it was made up of two particles, this could directly explain why light can be polarized [1].

Louis de Broglie was the first to elaborate a comprehensive theory on the possible internal structure of photons. According to his hypothesis as proposed in the 1930's, a permanently localized photon following a least action trajectory can satisfy at the same time Bose-

*Corresponding author: André Michaud, Senior Researcher, Canada, Tel : +1 418624 0608; E-mail: srp2@srpinc.org

Received October 17, 2015; Accepted Janaury 15, 2016; Published January 18 2016

Citation: Michaud A (2016) On De Broglie's Double-particle Photon Hypothesis. J Phys Math 7: 153. doi:10.4172/2090-0902.1000153

Copyright: ( 2016 Michaud A. This is an open-access article distributed under the terms of the Creative Commons Attribution License, which permits unrestricted use, distribution, and reproduction in any medium, provided the original author and source are credited. 
Einstein's statistic and Planck's Law, perfectly explain the photoelectric effect while obeying Maxwell's equations and totally conform to the properties of Dirac's theory of complementary corpuscles symmetry, only if it involves two particles, or half-photons of spin $1 / 2$, " that must be complementary with respect to each other in the same manner that the positive electron (the positron) is complementary to the negative electron in the Dirac Hole Theory" [2].

The following other quotes from the same reference summarize his hypothesis:

"Such a complementary couple of particles is likely to annihilate at the contact of matter by relinquishing all of its energy, which perfectly accounts for the characteristics of the photoelectric effect."

\section{Furthermore:}

"The photon, being made up of two elementary particles of spin $h / 4 \pi$, will obey the Bose-Einstein statistic as required by the precision of Planck's law for the black body."

Finally, he concludes that:

"this model of the photon allows the definition of an electromagnetic field linked to the probability of annihilation of the photon, a field that obeys Maxwell's equations and has all the characteristics of electromagnetic light waves."

Over the course of the 1930's and 1940's, de Broglie and his students progressively came up with an interesting and workable solution based on wave mechanics, that involved both corpuscles being singularities in an underlying wave phenomenon [1]. After Quantum Chromodynamics was developed in the 1970's an alternate model was developed, involving a mix of quark-antiquark pairs and gluons [3] based on this new theory and Quantum Mechanics, which also is an interesting and workable approach. A few other models have been proposed since, but all approaches have the similar downside with respect to Maxwell's theory of treating the electric and magnetic fields, either explicitly or implicitly, as a single "electromagnetic field" which turns out to be somehow featureless at the general level (the electromagnetic tensor), which distracts from permanent awareness that both fields are of equal and separate importance in Maxwell's theory, with different and irreconcilable characteristics, besides mutually inducing each other. This left no precise function being assigned to the "magnetic" aspect of the electromagnetic energy in a possible mechanics of mutual induction that would also involve the two separate charges, which are the "electric" components of the photon, a mechanics that would explain why photons can maintain sufficient local unity to account for their systematic and verifiable point-like behavior during scattering or absorption encounters, which includes all photons that we know have been emitted from the farthest reaches detectable in the universe, after having traveled for countless years. Indeed, the twin "electric" particles end up in both models as having an existence separate from the electric aspect of the electromagnetic energy that the localized photon is meant to represent, which introduces the required twin particles in a manner that does not incorporate them into the sequence of the electric vs magnetic mutual induction cycle that they theoretically are meant to enhance, according to de Broglie's initial hypothesis:

"it seemed to me that to obtain a clear image, in agreement with the classical concepts of the wave-particle dualism with respect to space and time, it was required to succeed in incorporating the particle into the wave" [1].

But it seems that the non-deterministic trend that was prevailing after the 1927 Solvey Congress confronted him with such difficulties that he ended up renouncing this ultimate goal [1]. Generalizing the electromagnetic interaction as a single tensor is a fine approach to obtain global perspectives, but it seems that looking for ever more detailed descriptions always favored deeper understanding of physical issues. This paper is then an attempt at exploring deeper even than the already interestingly detailed electric and magnetic fields as described by Maxwell's theory.

\section{The Required Internal Electromagnetic Symmetry}

As it stands, if the photon's double-component electric aspect is to remain coherent with its point-like behavior at the moments of emission and capture (or scattering), however long the time elapsed and distance covered between both events, the two separate "electric" half-photons have to unite in some fashion during each cycle of the photon's frequency to maintain point-like localization and most importantly to incorporate into mechanical process the other half of the electromagnetic relation, that is, its magnetic aspect. Doesn't the induction of an increasing magnetic field inseparable from changing current due to moving charges immediately come to mind at this point? In the case of photons, this brings displacement current into the picture, which would involve local motion of the postulated double charges that would cause the required change in the local electric field within the photon quantum, a current that would come into being without the presence of matter in this case, a process that interestingly was first proposed by Maxwell himself in 1865 and was the foundation of his electromagnetic theory [4] This in turn hints at the possibility of an internal oscillation of the photon energy related to its frequency.

Let's keep in mind here that the term "frequency" applies to any sort of cyclic motion, be it rotational, translational on a closed orbit or any other type of oscillatory motion, from simple sinusoidal harmonic motion to the cyclic translational reciprocating "swing" between two states being considered here and that we will term "oscillation" for simplicity's sake. This means that all aspects of angular momentum that we naturally associate with rotary motion can also be applied to reciprocating motion, which in turn allows the "spin" of elementary particles to be hypothesized as possibly corresponding to a reciprocating motion of the energy concerned without changing in any way the equations that already account for it.

It is a fact that all experimental research aimed at identifying charges in electromagnetic waves have failed to detect any in support of Maxwell's assumption. But let's consider that if electromagnetic waves as Maxwell conceived them really turn out to be only a convenient mathematical representation of a macroscopic perception of a crowd effect due to the presence of countless localized moving photons at the submicroscopic level, it would indeed be these individual photons that would display the searched for charges and would be the local sites of displacement current versus magnetic induction activity.

However, there exists no instrument sensitive enough to detect the infinitesimal fields of individual photons, with the added difficulties that they move at the speed of light and that any interception of a single photon simply incorporates its energy as an infinitesimal kinetic energy increment to one electron in one atom of the material that the detector is made of. But since this postulate was such a major and fruitful foundation in the elaboration of Maxwell's theory, which in turn allows such precise calculations, there seems to be no reason to do away with it now. The double-particle photon hypothesis would then imply that photons have to be stable localized moving electromagnetic structures whose energy quantum could logically only alternate between a two components electric state, with both components separating in space 
(an electric dipole), and a magnetic state involving only one component to explain permanent localization and that could consequently be dipolar in only one manner. Total symmetry of the magnetic aspect involving a single component can be obtained only if it consists in a single spherically expanding phase as both electric components move towards each other, followed by a spherical contraction phase as both electric components move away from each other; both magnetic expansion and contraction sequences of the magnetic component being normal to the electric phase at all times. This also means that the single magnetic component of the photon can be dipolar only along the time dimension since both expansion and contraction sequences cannot possibly occur simultaneously. Such a dynamic structure would still preserve the required fundamental symmetry since the spacewise moving electric dipole would be permanently counterbalanced by a related time-wise perpendicularly moving magnetic dipole, with both dipoles remaining perpendicular to the direction of motion of the photon in space, thus obeying the triple orthogonality required for plane wave treatment in Maxwell's theory's for straight line motion of electromagnetic energy.

\section{Internal Coulomb Interaction between the Half- photons}

Let us note here that de Broglie considered both half-photons as being electrically neutral [5] that is, not being charged negatively for one and positively for the other. But by the same token, he also discarded the possibility that Coulomb interaction could be involved in the process since he considered that the Coulomb force could be in action only between charged particles that are "signed" negatively and/ or positively, which was confirmed to me by his lifelong friend and colleague Georges Lochak, in correspondence initiated by me precisely to clarify this issue, which is why the research that he carried out did not take this possibility into consideration. Paradoxically, it has been understood and extensively experimentally confirmed since the 1930's that any photon of energy $1.022 \mathrm{MeV}$ or more, that has no rest mass and is electrically neutral, will destabilize and convert to a pair of electronpositron, massive and charged in opposition, when grazing a heavy particle such as an atomic nucleus. Could then the "signs" be an extrinsic property of elementary particles charges, possibly vectorial, that would be acquired during the separation process of the pair? This would leave the door wide open to the possibility that some form of Coulomb-like interaction might be involved at a level more fundamental than that of the acquisition of the opposite "signs" by the charges of the separating elementary particles. So let's dwell for a moment on what considering "signs" as a property separate from charges of elementary particles can allow visualizing. In this perspective, the very existence of "fractional signs" for the charges of the up and down quarks making up the inner scatterable structure of nucleons means that other "stable sign intensity levels" do exist besides the otherwise universal "unit sign intensity level" for the charges of electrons and positrons. Note that this comparison is by no means meant to hint at the possible origin of up and down quarks, which is still unresolved, but only to highlight the idea that different degrees of "sign intensity" do exist for stable particles, which allows considering that "sign intensity acquisition" for charges could possibly be progressive from null, for initially neutral photon charges, to maximum stable "unit sign intensity" for the charges of electron and positron, with intermediate stable levels corresponding to the up and down quarks' stable "fractional sign intensities". The opposite "unit sign intensities" of electron and positron could then be progressively acquired during the mother photon's destabilization process, possibly induced in the photon's initially neutral charges by the very presence of the "signs" of the charges of the destabilizing particle that the photon grazes, from neutral at the beginning of the process to maximum and stable opposite "unit sign intensity" for the separated charges if the destabilization sequence succeeds in separating the pair, or eventual regression back towards neutrality of the photon charges if the process fails for whatever reason, leaving the photon moving away with charges returning to neutral without decoupling for photons not energetic enough, or flying by too far from the destabilizing particle for the process to complete in the case of sufficiently energetic photons.

\section{Electrostatically Destabilizing Trajectories Intersections}

It must be considered also that Quantum Electrodynamics implicitly recognizes the presence of Coulomb interaction between a decoupling photon and a heavy nucleus, since it incorporates a Feynman's "virtual photon" into the pair production process representation (Figure 1), which was explicitly defined by Feynman himself as being a metaphor for Coulomb interaction [6], thus indirectly recognizing that Coulomb interaction has to be in action between the photon and the destabilizing heavy particle even before the pair separates, whatever the sign status of the photon's internal charges may have been. Let's consider what is likely to occur when a photon of energy $1.022 \mathrm{MeV}$ or more grazes very closely a heavy atomic nucleus. We know since de Broglie that all massive and charged elementary particles are electromagnetic in nature, since electric charges cannot be dissociated from a magnetic counterpart. This includes of course the scatterable point-like behaving massive up and down quarks making up the inner scatterable structure of nucleons (protons and neutrons) since they also possess measurable electric charges, charged quarks whose existence was not yet known when de Broglie was actively working on his hypothesis, since they were experimentally scattered against only in the late 1960's [7]. Destabilization leading to pair decoupling could then be explainable by the presence of these point-like behaving electromagnetic charged elementary particles of which all nucleons making up atomic nuclei are made, that can presumably enter into homo- and/or heterostatic interaction with the charges of the half-photons while the photon is in its electrostatic phase as it flies by. It becomes just as obvious then that these interactions may then become more and more intense in relation with the inverse square of the diminishing distance that separates the half-photons from these up and down quarks if a Coulomb-like law effectively applies, a process represented in Quantum Electrodynamics by the Feynman diagram shown in Figure 1 [8]. The fact that such decoupling can occur only during moments of very close proximity between photon and nucleus comes in support of the presence of an interaction as a function of the inverse square of the distance such as

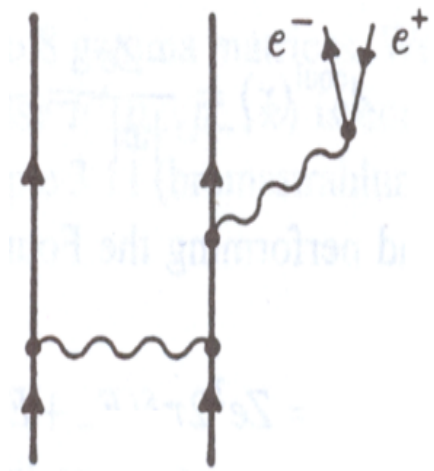

Figure 1: Photon-nucleus grazing pair creation Feynman diagram. 
the Coulomb law. Similarly, pair creation during close flyby of two photons, at least one of which exceeding the $1.022 \mathrm{MeV}$ minimum energy threshold without any atomic nuclei being close by, such as was first experimentally confirmed by Kirk McDonald et al. at the Stanford Linear Accelerator in 1997 with experiment \#e144 [9], is represented by the Feynman diagram shown in Figure 2 [8]. So there seems to exist sufficient supporting evidence to at least explore the possibility that Coulomb-like interaction could be at play between photons and other localized electromagnetic particles and even between the possibly neutral charges of the de Broglie double-particle photon.

\section{Photons, Electrons, Positrons, Exclusively Made of Kinetic Energy}

After destabilization, the separated halves of the photon's energy can thereafter be observed behaving as one massive $0.511 \mathrm{MeV} / \mathrm{c}^{2}$ electron plus one massive $0.511 \mathrm{MeV} / \mathrm{c}^{2}$ positron traveling separately, whose unit charges are now observable as being signed in opposition, and whose velocity away from each other is linked to the residual energy that the mother photon possessed in excess of the $1.022 \mathrm{MeV}$ energy threshold level which is now making up the rest masses of both particles, a process first observed and confirmed by Blackett and Occhialini from analyzing recorded cosmic radiation scattering impact traces in a bubble chamber in the early 1930's. The reverse process of electron-positron pairs re-uniting to entirely convert back to various photon states has also been first observed and confirmed by Blackett and Occhialini, such as in the case of positronium decay. So both reverse processes constitute the de facto irrefutable material proof that electrons and positrons are made of the very same energy and are of the very same electromagnetic nature as photons. In addition to this process of massive electron-positron pairs converting back to free moving electromagnetic energy photon state, we know that electromagnetic photons are created in a variety of other circumstances. But on final analysis, they all turn out to involve the emission of an electromagnetic photon when a charged particle, such as an electron, is suddenly stopped in its motion towards the oppositely signed nucleus of an ionized atom, for example, or similar processes involving metastable partons or events inside nuclei. If we take the process of a photon being emitted as an electron is being captured by an ionized atom for example, the photon that then escapes verifiably carries away part or all of the kinetic energy that the incoming electron was initially endowed with, if any, plus the additional kinetic energy that it accumulates during its Coulomb force related freefall acceleration towards the location of its brutal relative stop en route towards the attracting atomic nucleus, a location where it is captured in some

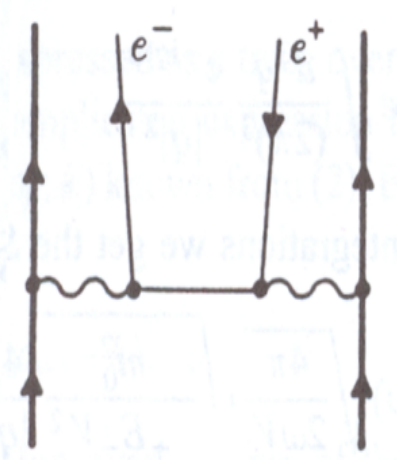

Figure 2: Photon-photon flyby pair creation Feynman diagram. overwhelming local electromagnetic equilibrium state on some allowed orbital about the nucleus, where it is left with only the exact amount of energy allowed in this new equilibrium state, an amount related to the distance now separating it from the oppositely signed nucleus. Besides this case of free moving electrons being captured by ionized atoms, the other familiar cases involve electrons having moved further away from a nucleus after having been momentarily excited to a metastable higher energy state, that go back to a lower energy state as they return to an orbital closer to the nucleus, where a photon is emitted to release the kinetic energy that now becomes in excess for this closer location. This motion of an electron being momentarily sufficiently excited to move to a metastable orbital further away from an atomic nucleus, or to outright completely escape from the atom, is always due to this electron having been excited away from its rest orbital through conduction or convection transmitted kinetic energy when in gaseous, liquid or solid materials, or having absorbed a discrete amount of kinetic energy from being collided with by an incoming photon, the latter sometimes being completely absorbed in the process, sometimes relinquishing only part of its energy and moving on with the remainder as a less energetic photon, such as in Compton or Raman scattering.

Photons can thus carry away a variety of discrete amounts of kinetic energy depending on local circumstances, whose individual frequencies cover the complete gamut of the electromagnetic spectrum, from the longest radio wavelengths to the shortest gamma wavelengths, the latter due to similar emission processes at the level of atomic nuclei. The whole collection of these photons is of course what allows us to see the universe as they hit the sensory cells in our retinas and/or the sensors of our instruments, allowing us in turn to observe and understand our surroundings up to and including determining the composition of stars. The process of kinetic energy accumulation by charged particles during Coulomb force induced freefall acceleration can easily be verified experimentally at our macroscopic level in a number of ways; with Coolidge tubes for example, as photons are liberated carrying away the exact amount of kinetic energy accumulated during the acceleration phase between the electrodes by electrons that suddenly come to a brutal stop (bremmsstrahlung) as they are captured by ionized atoms located on the anode (or anti-cathode). Emission of photons due to sudden stop of accelerating particles can also be verified with electron beams that are magnetically wiggled in particle accelerators, submitting the electrons in the beam to cyclic transverse accelerations and slowing-downs as the beam is forced to oscillate from side to side, producing so-called synchrotron "radiation", typically in the X-ray range; or in high energy accelerator storage rings, where beams of charged particles are repeatedly forced by magnetic pulses to maintain a best fit approximately circular trajectory.

Now, the issue always remained unclear as to how unidirectional kinetic energy (aka "translational energy") accumulating through acceleration of massive and charged particles can "become" electromagnetic when it is liberated as a photon. Let us recall that the electric and magnetic "fields" of Maxwell's theory are only mathematical representations meant to allow us to describe the observed behavior of electromagnetic energy, which is physically existing in objective reality.

Indeed, there is no prima facie reason for this unidirectional kinetic energy to change in nature during the various processes that we examined, particularly since we directly recuperate it as the plain unidirectional kinetic energy that first apparently "converted" to photon state when a bremmsstrahlung photon is being "emitted" by an electron, or when a mother photon's residual energy in excess of the $1.022 \mathrm{MeV}$ going into the rest masses of a separating pair, is observed 
precisely defining the velocity away from each other of both particles as unidirectional kinetic energy. If kinetic energy does not change in nature during these various processes, this also possibly means that what we perceive and measure as "charges" could also be a relative property that could become perceivable only as the unidirectional kinetic energy is in the process of separating to escape as a free moving photon, just like the opposite signs of isolated massive particles (electron and positron) could be relative properties that would be acquired as the particles come into being when the mother photon decouples.

So, let us then keep in mind as we move on that we will be attempting to explain how and why discrete quanta of this intriguing "substance" that we name "kinetic energy" can possibly move freely at the speed of light as discrete "electromagnetic" quantities without changing in nature. It doesn't seem unreasonable either to think that this "substance" that we identify as "kinetic energy" may have some form of "physical presence", since its quantized manifestations (photons, electrons, positrons, for example) can verifiably be mutually scattered against each other. Before proceeding further, let's define more precisely what "physical presence" could mean in the present context. We do not know and may never know what this "substance" or "fluid" really exactly is that we name kinetic energy. It may be possible however to come to terms with a usable "nearest possible approximation" of what its physical presence could be. De Broglie on his part thought of electromagnetic energy in terms of a "virtual fluid" [1].

"If we suppose known the form of the wave linked to a particle, the intensity of this wave at each point and at each instant (given by $|\psi|^{2}$ ) can be considered as defining the density of a virtual fluid (un fluide fictif) moving in space as time progresses and then the quantity of this fluid contained in an element of volume will give the probability for the particle to be present within this element of volume."

We will be going one step further here considering the apparent identity that seems to exist between fundamental electromagnetic energy and unidirectional kinetic energy that accumulates by means of acceleration, if the latter does not change in nature during the various changes of state that we examined. If we consider a rotating fan for example, there is no doubt that the incompressible volume of space cyclically visited by the rotating blades can be measured and studied, even though we know that the actual volume occupied by the material making up the blades and the nature of this material have no relation at all with the incompressible volume that the moving blades visit. If the blades of this fan were invisible to us and if we had no idea even of their existence, we nevertheless could study and measure the incompressible volume that the invisible rotating blades cyclically visits, due to the simple fact that trying to touch that volume would have physical consequences that we could then measure and that would allow us to try ascertaining its properties. We would be left to wonder however, forever maybe, at what could be causing this volume to exist at all with the possibly unexpected properties that our measurements seem to reveal. Indeed, how could we ever discover the existence of the blades and the nature of the material that they are made of, given that no clue to any of their characteristics are given by our measurements?

We find ourselves in a similar predicament regarding the possible "physical presence" of kinetic energy. We can possibly measure the physical presence of a "volume" for kinetic energy and assign to it the properties required to explain its observed behavior, even though this may not reveal the actual real cause and real nature of what is causing this "volume" to exist. For the needs of the present analysis, properties such as incompressibility, fluidity, and elasticity could tentatively be assigned to this "volume", to describe the tendency of the energy that resides in this volume to always remain in motion within this volume as the electromagnetic oscillation suggests, and/or alternately to also constantly tend to move in straight line in space when external electromagnetic equilibrium is not restraining it.

So let's proceed with this tentative "nearest possible approximate definition" for some form of "physical presence" of kinetic energy for the moment, within the frame of the state of our current knowledge about electromagnetic energy, subject to correction or completion as required. Now, if kinetic energy doesn't change in nature as it quantizes as free moving photons, the internally oscillating motion of the kinetic energy quantum could metaphorically be immobilized. The energy of this quantum could then be theoretically reduced to the smallest spherical uniformly isotropic volume that it could occupy, for the purpose of assessing its absolute density. This volume, that could be named the theoretical stationary isotropic volume of the energy of a photon, however small, would depend on the local amount of this kinetic energy and could then be calculated ([10], equations (40) to (41)). We will use this volume in equations (31) to (36).

\section{The fundamental question can now be summarized as follows:}

How can a quantity of kinetic energy, accumulating due to Coulomb force freefall acceleration of a massive particle (an electron for example) as the latter unidirectionally increases its velocity in space to start with, dynamically "fold" onto itself according to the threefold orthogonal relation revealed by Maxwell's theory, to become a stable quantum of electromagnetic energy escaping at the speed of light (a photon), while being animated with the local multidirectional oscillating motion suggested by de Broglie's hypothesis; a quantum whose energy would consist in a space-wise electric dipole cyclically morphing into a time-wise magnetic dipole, and that could also explain all electromagnetic properties of photons without changing in nature?

It must be obvious at this point that all photons have to be made of the same material, that is, quantized amounts of kinetic energy, an apparently physically existing "substance" that we still know so little about and that appears to be the only "material" of which all photons and all existing charged and massive elementary particles seem to be made of.

\section{The Distribution of Kinetic Energy within a Localized Photon}

Now, the question comes to mind as to how this kinetic energy organizes within the photon to sustain an electromagnetic oscillation at a particular frequency and at the same time sustain its own motion at the speed of light.

Clues to this internal structure were given by a brilliant analysis carried out by Paul Marmet in an article that was accepted for publication in the Kazan State University International IFNA-ANS Journal, in 2003, titled: "Fundamental Nature of Relativistic Mass and Magnetic Fields" [11]. His analysis of the relation between the relativistic magnetic mass increase of a moving electron in relation to relativistic velocities allowed defining an LC equation that can describe a possible dynamic internal energy structure for the carrying energy of the electron in motion. In turn, this LC equation allowed upgrading Newton's non-relativistic kinetic equation $\mathrm{K}=\left(\mathrm{mv}^{2}\right) / 2$ to relativistic status [12]. It is the observation that the speed of light is obtained when the mass of the electron is set to zero in this relativistic equation, leaving behind only the carrying energy, that finally reveals that free moving electromagnetic photons (carrying no massive particle) are likely to 
have the same internal electromagnetic LC structure as that of the carrying energy of moving electrons. Marmet obtained the following definition of current by quantizing the charge, which removed the time element from the equation as he replaced $d t$ by $d x / v$, since the velocity of current is constant at any given instant:

$$
I=\frac{d Q}{d t}=\frac{d(\mathrm{Ne})}{d t}=\frac{d(\mathrm{Ne}) v}{d x}
$$

Where $e$ represents the unit charge of the electron and $N$ represents the number of electrons in one Ampere. Substituting the resulting value of $I$ in the scalar version of the Biot-Savart equation then allowed doing away with the time element in this equation also:

$$
d \mathbf{B}=\frac{\mu_{0} I}{4 \pi r^{2}} \sin (\theta) d x=\frac{\mu_{0} v}{4 \pi r^{2}} \sin (\theta) d(\mathrm{Ne})
$$

Without going into the detail of his derivation, which is very clearly laid out in his paper ([11], Equations (1) to (26)), let us only mention that the final stage of this development consists in spherically integrating the electron magnetic energy, whose density is mathematically deemed to vary from a minimum limit corresponding to $r_{e}$ to a maximum limit located at infinity.

$$
M=\left\{\frac{\mu_{0} e^{2} v^{2}}{2(4 \pi)^{2} c^{2} r^{4}}\right\} 2 \pi \int_{0}^{\delta} \sin (\theta) d \theta \int_{r_{e}}^{\infty} r^{2} d r
$$

The electron classical radius $r_{e}$ is the mandatory lower limit in such an integration to infinity, due to the simple fact that integrating any closer to $r=0$ would accumulate more energy than experimental data warrants. After integrating, he obtained:

$$
M=\frac{\mu_{0} e^{2} v^{2}}{8 \pi r_{e} c^{2}}=\frac{m_{e}}{2} \frac{v^{2}}{c^{2}}
$$

which very precisely corresponds to the total mass of the magnetic field of an electron moving at velocity $v$. He discovered by the same token that any instantaneous "magnetic mass" increase of an electron is a direct function of the square of its instantaneous velocity.

When this velocity is small with respect to the speed of light, the following classical equation is obtained, allowing clearly determining the contribution of the magnetic component to the rest mass of the electron:

$$
\frac{\mu_{0} e^{2}}{8 \pi r_{e}} \frac{v^{2}}{c^{2}}=\frac{m_{e}}{2} \frac{v^{2}}{c^{2}}
$$

Where $r$ is the classical electron radius (2.817940285E-15 m), and $e$ is the charge of the electron (1.602176462E-19 C), and from which can be concluded that the invariant magnetic component of the electron at rest corresponds to a mass of:

$$
M_{0}=\frac{\mu_{0} e^{2}}{8 \pi r_{e}}
$$

which is exactly half the mass of an electron, the other half being made up of what could be termed its "electric mass", since the electron is an electromagnetic particle. Paying attention to the difference between equations (4) and (6), we observe that $M-M_{0}$ represents the relativistic mass increment related to instantaneous velocity $v$. We note also that the translational kinetic energy required to propel the electron at this velocity is absent from the equation. Close analysis and calculation reveals however that the amount of translational kinetic energy required to propel an electron with magnetic mass $M$ at velocity $v$ is exactly equal to the amount of energy captive in the instantaneous relativistic mass increment $M-M_{0}$.
This means that the total amount of energy that must be communicated to an electron at rest for it to move at any velocity must be defined as an amount of translational kinetic energy plus an equal amount of kinetic energy that momentarily converts to the instantaneous relativistic mass increment related to that velocity.

$$
\mathrm{E}_{\text {total }}=\mathrm{E}_{\text {translational }}+\mathrm{E}_{\mathrm{r}}
$$

Since energy in motion cannot be dissociated from electromagnetism, it can be surmised that an electric component is de facto involved in relation with the half of the energy that in context clearly is "magnetic" in nature, and the only way it can be introduced in context is for this magnetic energy to alternate between this magnetic state and an electric state at the frequency that can be associated to this amount of energy.

$$
\mathrm{E}_{\text {total }}=\mathrm{E}_{\text {trans. }}+\left[\mathrm{E}_{\text {elec. }} \cos ^{2}(\omega t)+\mathrm{E}_{\text {mag. }} \sin ^{2}(\omega t)\right]
$$

This form in turn immediately suggests the following LC relation to represent the internal structure of the carrying energy of an electron in motion:

$$
E=\frac{h c}{2 \lambda}+\left[\frac{e^{2}}{2 C_{\lambda}} \cos ^{2}(\omega t)+\frac{L_{\ddot{e}} i_{\lambda}^{2}}{2} \sin ^{2}(\omega t)\right]
$$

where $\lambda$ is the wavelength associated to this amount of electromagnetic energy in motion and where the following are the classical equations for calculating capacitance and inductance during a LC cycle:

$$
E_{\mathbf{E}(\max )}=\frac{q^{2}}{2 C} \quad \text { and } \quad E_{\mathbf{B}(\max )}=\frac{L i^{2}}{2}
$$

Equation (9) reveals that all probabilities are that the velocity of light of an isolated electromagnetic photon would be maintained because the translational half of its kinetic energy serves to propel at this velocity an equal amount of kinetic energy while the latter permanently oscillates between an electric state and a magnetic state at the frequency determined by the total amount of kinetic energy involved. This structure will be analyzed in detail further on.

\section{The Neglected Classical Maxwellian Space Geometry}

Maxwell's theory is traditionally considered from the mathematical viewpoint offered by his famous equations and understood within the restrictive perspective of plane wave treatment, leaving the space geometry that underlies it to be mostly taken for granted, since it is sufficient for the needs of the continuous wave concept, which in turn is sufficient for precise calculations at the general level. This classical space geometry is of course the traditional Euclidian 3-dimensional flat space geometry to which the time dimension is added to justify motion. Just like the habit of using the electromagnetic tensor to represent a single "electromagnetic field" concept keeps away from immediate attention that both electric and magnetic fields are of equal and separate importance in Maxwell's theory, with different and irreconcilable characteristics, the habit of using plane wave treatment leaves in the background the fact that the wave front of the electromagnetic wave of Maxwell's theory could only be in spherical expansion from some point-like source, a point-like source which is confirmed out of any doubt by experimental reality for any electromagnetic quanta emission, even if Maxwell's continuous waves had been proven to really exist. Maxwell's theory is in fact the natural end result of the integration of many discoveries made previously. His first equation is In Gauss' law for electricity; his second equation is derived from Faraday's law, his third from Gauss' law on magnetism and his forth is a generalization of Ampere's law. What Maxwell did in fact was unify into one coherent 
integrated theory all these experimentally confirmed laws that were not clearly linked to each other previously.

But his really brilliant personal contribution was his success in mathematically linking Faraday's law and his modified Ampere's law in such a way that no doubt could remain that light was intimately linked to electricity and magnetism, as confirmed by Faraday's experiments on light polarization by magnetic fields. Linking them provided as a side benefit the only way ever devised to calculate light velocity from first principles, a velocity that is the only velocity possible from these equations since it rests on the products of only two other fundamental constants, that is, the electric permittivity and magnetic permeability constants of vacuum. As already mentioned, a fundamental and thoroughly verified aspect of Maxwell's theory is the mandatory state of orthogonality that must exist between the electric and magnetic fields of free moving electromagnetic energy, both fields also being normal to the phase velocity vector that identifies the direction of motion of any point considered on the wave front of the spherically propagating "wave". Experimental reality reveals that this triple orthogonality also applies to the motion of charged massive particles, such as electrons being forced to move in straight line when subjected to equal density external electric and magnetic fields. Indeed, any elementary textbook on electricity and magnetism explains how the vectorial cross product of equal intensity electric and magnetic fields being applied to a charged particle will generate a velocity vector in straight line forcing the particle to move in a direction perpendicular to both resulting forces. The more intense the fields, the faster the particle will move, and whose varying velocity is given in classical electrodynamics from the Lorentz equation, by this well known relation:

$$
\frac{\mathbf{E}}{\mathbf{B}}=v
$$

Which resolves to the fixed speed of light " $\mathrm{c}$ " for photons, from Maxwell's 4th equation:

$$
\frac{\mathbf{E}}{\mathbf{B}}=c
$$

Or rather, in the present context, under the form of a vectorial cross product:

$$
\mathbf{E} \hat{j} \times\left(\frac{-1}{\mathbf{B}}\right) \hat{k}=\mathbf{E}\left(\frac{-1}{\mathbf{B}}\right) \cos \theta \hat{i}
$$

and since angle $\theta$ must be equal to $90^{\circ}$ by definition in the case of the straight line motion that we are considering:

$$
\mathbf{E} \hat{j} \times\left(\frac{-1}{\mathbf{B}}\right) \hat{k}=v \hat{i}
$$

where $v$ is the velocity vector.

The orthogonal bases shown in Figure 3 will be used in this paper:

a) $3 \mathrm{D}$ rectangular $\mathrm{x}-\mathrm{y}-\mathrm{z}$ coordinate system, and corresponding rectangular unit vectors base and

b) The correspondingly oriented rectangular electromagnetic fields vs velocity vector base:

It is generally understood also that despite the precision of the calculations that Maxwell's theory allows for electromagnetic energy, his theory is deemed unable to directly describe photons as discrete localized moving electromagnetic particles since it is grounded on the notion that electromagnetic energy is a continuous wave phenomenon.



Figure 3: Orthogonal bases used in this paper.

\section{Discrete Particles as the Only Possible Support of Electromagnetic Properties}

Maxwell's theory, as a matter of fact, was designed to account for electromagnetic energy behavior at the macroscopic level without the need to take quantization into account which had not yet been clarified in Maxwell's time, that is, by treating electromagnetic energy as a featureless energy density per unit volume or featureless energy flow per unit surface rather than by adding the energy of localized moving electromagnetic photons enclosed in a unit volume or flowing through a unit surface, that would take localization into account and would represent just as well all observed electromagnetic phenomena at the macroscopic level. Considering that the "electromagnetic waves" that Maxwell conceived of were meant to animate what was perceived from our macroscopic level as a still hypothetical underlying and all pervading "ether", then if some means was found to associate to each individual localized photon all of the electric and magnetic properties that characterize the electromagnetic wave of Maxwell's theory, this would remove the theoretical need for the existence of such a supporting all pervading medium for the purpose of supporting continuous electromagnetic waves, that we now know do not exist at the submicroscopic level. Let us note also that a second theoretical use of the various forms of the concept of ether was for it to constitute the very substance that massive particles were made of as "singularities" that developed in such all pervading ether fields in a variety of theories. Now if kinetic energy, of which discrete localized photons are demonstrably made, turns out to have "physical presence" with a "volume" that can be measured, this would altogether remove the last reason that would justify resorting to the theoretical concept of ether as a basis to explain the fundamental level of physical reality. All the more so since it has been conclusively verified since the 1930's that massive electron and positron can be made from destabilizing electromagnetic photons containing at least $1.022 \mathrm{MeV}$ of this kinetic energy [13]. Head-on collision experiments between beams of electrons and positrons [14] even lead to suspect that protons and neutrons could be stable adiabatic equilibrium states involving triads of electrons and positrons that could have interacted in such a way that they could have locally adiabatically accelerated until they reach these two ultimate and irreversible equilibrium bound states [15]. Of course, such a possibility seems at first glance to be in total contradiction with the Principle of conservation of energy. But considering that all existing closed systems for which the Principle of conservation of energy can be verified to apply have already reached some form of least action energy equilibrium, that can be modified only by introducing energy in excess of this equilibrium, there exists the possibility that newly created particles, that never were chased out of some pre-existing least action equilibrium state, could accumulate new energy by means of an initial 
and irreversible adiabatic acceleration process that would bring them to such a first least action energy equilibrium state, after which they would of course be forever subjected to the Principle of conservation. We must not forget either that even if ether could finally be done away with, more and more data seems to indicate that here on Earth, we are permanently immersed in an all pervading interacting magnetic fields combination involving the Earth's magnetic field moving through the immense magnetic field of the Sun that reaches way beyond Pluto, which also interacts with the local magnetic fields of the other planets of the Solar system, and finally there seems to be little doubt that the global magnetic field of our local galaxy also interacts with the Sun's magnetic field.

So, whatever the final solution will be, it will mandatorily involve this all pervading underlying medium in what we consider as the total vacuum of space.

\section{The Issue of Intensity Conservation with Maxwell's Spherically Expanding Wave Concept}

This leads to attempt clarifying why an acceptable description of electromagnetic photons as stable permanently localized moving particles, in line with their demonstrated point-like localization at the moments of emission and capture, more than one century ago, has not yet been successfully reconciled with the verified aspects of Maxwell's theory, particularly after Louis de Broglie elaborated his intriguingly promising hypothesis [2]. According to Maxwell's theory, the electric and magnetic aspects of an electromagnetic wave must by necessity always be in phase at the wave front (Figure 4), that is, at maximum at the same moment, for the wave to exist at all and propagate. When both aspects are $90^{\circ}$ out of phase, we obtain a standing wave (Figure 5). But as an intriguing dead end in Maxwell's theory, when both aspects are set $180^{\circ}$ out of phase, we end up with the exact equivalent of both aspects being in phase (Figure 4), But we will see further on that far from being a dead end in physical reality, this $180^{\circ}$ dephasing will turn out to be in perfect harmony with the LC oscillation for which we will give the mathematic development (Figure 5). Also, it is the conjunction of both fields, in phase and at right angle with each other at all points of the wave front that is deemed to maintain the intensity of the energy of the wave at every points of the wave front, despite the inherent spherical spread involved from the mandatory point-like origin of such a wave, if it really existed. This issue is of course familiar to all but is apparently seen as an unavoidable axiom, no doubt resting on the comfortable fact that plane wave treatment allows precise calculation anyway. Mathematically speaking, when any point of the curved spherical wave front surface is considered, this surface can be locally approximated

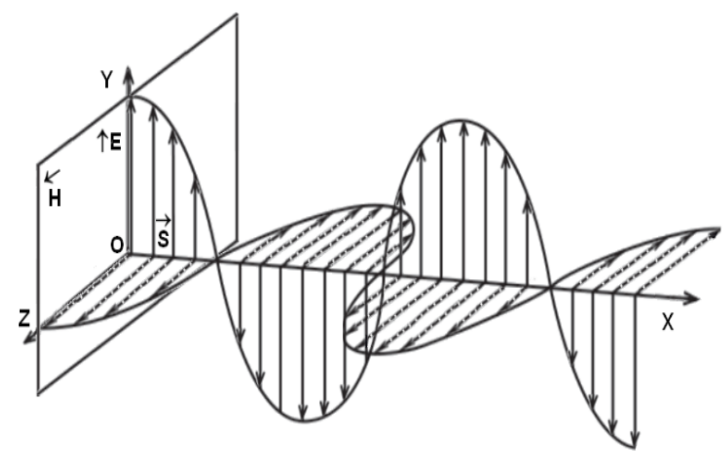

Figure 4: Electric and magnetic fields in phase, or $180^{\circ}$ out of phase, in classical electromagnetism.

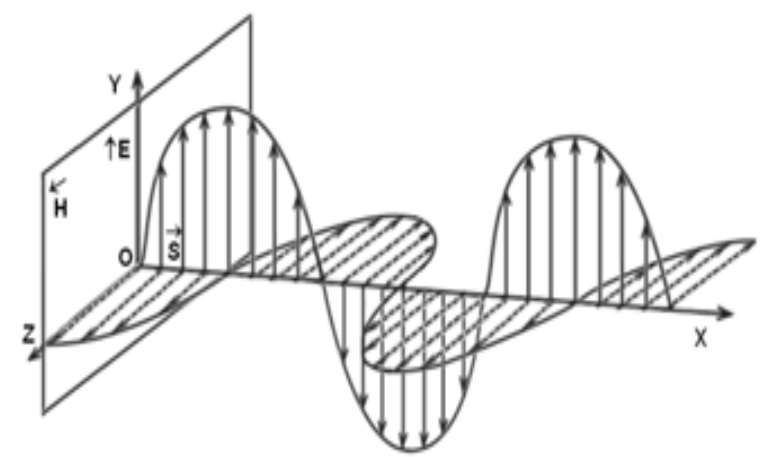

Figure 5: Electric and magnetic field 900 out of phase in classical electromagnetism.

to a plane surface at the infinitesimal level which is the origin of the "plane wave" equations set. But space being three-dimensional, such treatment with the plane wave analogy can only be a mathematical approximation obscuring the fact that if such an electromagnetic wave really physically existed, it could only be in spherical expansion from its initial point-like state, assuming unbounded isotropic expansion. So plane wave treatment applied to Maxwell's theory currently does not describe electromagnetic energy as it starts existing at its pointlike source, but only after the expanding wave has begun to propagate. Also, the geometry of such a spherically propagating wave would be much more similar to the spherical expansion of a sound wave from its point-like source in some underlying medium than to the propagation of waves on a plane liquid surface that immediately comes to mind when thinking about plane wave treatment. It then becomes very difficult to accept the idea that the initial intensity of the point source of the wave could be arbitrarily multiplied in such a way that it could be measured as equal to the energy of the source at any point of the expanding spherical wave front at any arbitrary distance from the punctual source, as plane wave treatment seems to allow. So the habit of dealing with the state of orthogonality of both fields with respect to each other and to the direction of motion in space of any point on the already expanding wave front always leaves in the background the fact that such a spherically expanding wave can only be a single electromagnetic event originating from a single point-like source.

\section{Applying Electromagnetic Properties to Maxwell's Spherically Expanding Wave's Point-like Initial State}

Now, considering that such an electromagnetic event is a single event, could it not be imagined that after appearing at its point-like origin, it could be represented as remaining locally point-like as it starts moving, harmonically oscillating as it moves, which is what de Broglie's hypothesis implies, instead of spherically expanding as Maxwell's theory implies by definition?

This would involve a precise trajectory being followed by this electromagnetic event, which would then behave point-like from emission to capture, which would in turn be in total harmony with the verified fact of its point-like capture, however much time could have elapsed after it was emitted and whatever distance it could have covered before being captured. This would also directly explain why the initial intensity of this electromagnetic quantum is conserved, barring energy losses or gains through red or blue shifting due to gravitational interaction along the path that it would have followed. The idea naturally comes to mind then that the state of fundamental orthogonality of both electric and magnetic fields could possibly be served just as well, 
if not better, by being defined with respect to the electromagnetic event immediately as it initially appears point-like, instead of after spherical expansion is already under way. But the apparently insurmountable issue with this approach in classical electrodynamics is the mathematically assumed infinite energy associated with such a punctual electromagnetic concept.

Another problematic issue comes to light with the idea of mathematizing free moving energy at its point-like source. It is the fact that both fields of any point-like electromagnetic quantum (a photon) which is in the process of being emitted by a de-energizing electron can be orthogonal to no particular direction in space at the very moment of point-like separation, which leads to the conclusion that at the very moment of separation, both fields of the new photon could be orthogonal only to 3D space proper, despite the strangeness of the idea.

Considering also that electric interaction obeys the inverse square law of electrostatic attraction and repulsion between the charges of elementary particles and that magnetic interaction obeys the inverse cube law of magnetostatic attraction and repulsion between magnetic fields of the same elementary particles, makes it appear illogical, and even impossible, that quantized quantities of kinetic energy could possess both electric and magnetic properties at the same moment, or even in alternance, while not changing in nature.

The inverse square interaction law between electrically charged elementary particles, that is, the Coulomb law, is very familiar, but the inverse cube law between the magnetic aspects of the same point-like behaving elementary particles is much less familiar. A direct confirmation of this inverse cube relation has been very recently obtained by Shlomi Kotler and his team between the magnetic aspects of two electrons, as reported in the Nature magazine in April 2014 [16] thus confirming by the same token the validity of the lab bench experiment carried out 15 years ago which is described in reference [17].

It is precisely the combination of these mutually incompatible inverse square law applying to the electric aspect of a point-like behaving particle and of the inverse cube law applying to its magnetic aspect that elicits the strongest doubt on the ability of classical 4D spacetime geometry to allow the kinetic energy that the particle is made of to continue displaying these irreconcilable properties while not changing in nature as it electromagnetically oscillates while moving at the speed of light through vacuum, if kinetic energy is to be considered being a "physically existing substance". These considerations are what gave birth to the idea that the physically existing space geometry at the fundamental level may be more complex than can be directly observed from our macroscopic level, and that extra "spaces" could possibly be involved to allow for these possibilities, that is, a second space that would allow kinetic energy to display electric characteristics without changing in nature and a third space that would allow kinetic energy to display magnetic characteristics without changing in nature, both extra spaces remaining permanently perpendicular to each other and to normal space at the particle level.

It is to be noted at this point that Louis de Broglie also came to the conclusion from other considerations that it was impossible to exactly represent elementary particles in the restricted frame of continuous three dimensional space.

"The non-individuality of particles, the exclusion principle and exchange energy are three intimately related enigmas; all three are tied to the impossibility of exactly representing elementary physical entities within the frame of continuous three dimensional space (or more generally of continuous four dimensional space-time). Some day maybe, by escaping from this frame, will we better grasp the meaning, still quite cryptic today, of these major guiding principles of the new physics" [2].

An expanded space geometry that allows a clear definition of the double-particle photon without its kinetic energy quantum changing in nature, and that may also allow resolving some of the issues raised by de Broglie was first introduced in July 2000 at Congress-2000 at St. Petersburg State University [18]. This new space geometry will now be described before proceeding to build the LC and local fields equations that can represent the permanently localized double-particle photon in this expanded space geometry.

\section{Expanding Space Geometry beyond Normal 3D Space}

As previously done with the idea of a usable "nearest possible approximate definition" for the "physical presence" of kinetic energy as a "physically existing substance", we may think of this expanded space geometry as a usable "nearest possible approximate definition" of the required space geometry, within the frame of the current state of our knowledge about electromagnetic energy. If we imagine the observed electric behavior of charges as being due to the momentary presence of the incompressible energy of a photon in a separate 3D-space that allows such behavior, and magnetic behavior as being due to the alternate momentary presence of the same energy in a different 3D-space that allows such behavior, each space being governed by the same laws of motion as normal 3D-space, the same capacitance and inductance, both spaces remaining permanently perpendicular to each other and to normal space, and that would allow the circulating kinetic energy not to change in fundamental nature, it will become possible to visualize much more clearly the internal oscillation of the kinetic energy of the localized double-particle photon of de Broglie's hypothesis.

In order to more easily refer to these new spaces, let us name electrostatic space the space into which kinetic energy displays electric behavior, and magnetostatic space the space into which it displays magnetic behavior. For coherence, we will identify normal, electrostatic and magnetostatic spaces as being X-space, Y-space and Z-space respectively. Within normal space, let us rename the three minor spatial dimensions: $\mathrm{X}-\mathrm{x}, \mathrm{X}-\mathrm{y}$ and $\mathrm{X}-\mathrm{Z}$ and likewise, for electrostatic and magnetostatic spaces: $Y-x, Y-y, Y-z$ and Z-x, Z-y, Z-z. Let us assume furthermore that the minor $x$-axes of all 3 spaces are mutually parallel in a direction corresponding to the conventional direction of motion of energy in normal space in plane wave treatment. Of course, when the $\mathrm{x}, \mathrm{y}$ and $\mathrm{z}$ dimensions are used without major axis prefix, they refer as usual to normal 3D space. In this space geometry, a point-like junction (representing a "passage point" in physical reality, not really a dimensionless "point" in the mathematical sense, whence the best-fit representation phrase "point-like" being used, which does not exclude the possibility of a local "volume" or "area", however small, being involved) between these three orthogonal spaces would be located at the geometric center of each photon, and it is this point-like junction that would be moving point-like at the speed of light in normal X-space, that is, along the $\mathrm{X}-\mathrm{x}$ axis of this expanded geometry in plane wave treatment (Figure 6).

To be able to mentally visualize the locally standing motion of kinetic energy in this 3-spaces structure, an easily mastered technique can be use. It suffices to imagine the 3 familiar minor $x-y-z$ orthogonal dimensions of normal 3D space as if they were the ribs of an open 3-ribs metaphorical umbrella, the apex of which would be located at the origin (or passage point where the 3 spaces meet). If we mentally fold the umbrella, we can now visualize the folded umbrella as if it was the linear major $\mathrm{X}$-axis of this expanded coordinates superset. With 


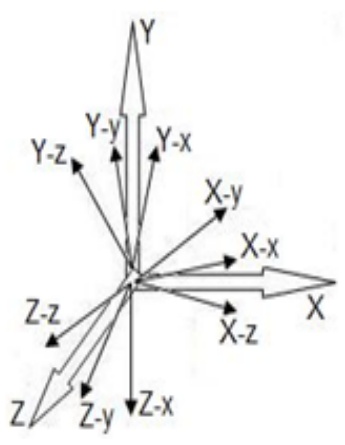

Figure 6: The orthogonal structure of the 3-spaces model.

this umbrella metaphor, it is now easy to visualize the three orthogonal spaces as three umbrellas meeting at their tips. We only need to mentally open any one of them to examine what is occurring in this particular space at any given moment of the electromagnetic cycle. As observed from within normal space, which will be our observer's viewpoint during this analysis, free fall acceleration induced unidirectional kinetic energy accumulating within the same normal space will be locally perceived as having longitudinal inertia but no transverse inertia. The longitudinal inertia of electromagnetic photons was experimentally confirmed more than one century ago by the same photoelectric proof due to Einstein that confirmed that electromagnetic energy behaves as discrete localized quanta and not as a continuous wave phenomenon. The absence of transverse inertia for unidirectional kinetic energy on its part was experimentally demonstrated more than one century ago also by Walter Kaufmann [19] as he demonstrated that the transverse inertia of electrons accelerated to relativistic velocities was lower than their longitudinal inertia. This issue will be analyzed further on. From within normal space again, all energy present within electrostatic and magnetostatic spaces at any given moment of the electromagnetic cycle will appear to possess both longitudinal and transverse inertia, that is, omnidirectional inertia; in other words: electromagnetic mass. Metaphorically speaking, the energy present in these two extra spaces would appear to be captive inside some invisible "container" that will resist being pushed around from any direction from within normal space. Although photons are known not to have a rest mass, they are also known to possess an electromagnetic mass that can interact gravitationally. The photon itself will now appear as a discrete amount of kinetic energy, half of which remaining unidirectional and moving in normal space, as determined in Section 6, propelling the other half that would be oscillating cyclically through the point-like junction between electrostatic and magnetostatic spaces at the frequency determined by the photon's energy. A separate analysis explains why half of any localized photon's kinetic energy (that is the photon's translational energy) has no option other than to remain unidirectional within the photon's inner structure, even without invoking the 3-spaces concept nor the double-particle concept, to propel the other half of a localized photon's energy at the speed of light [12]. A property of unbounded elasticity and fluidity for the kinetic energy "substance" can even allow for both half-photons to possibly not be "completely severed" from each other nor from the portion moving unidirectionally in normal space as they separate within electrostatic space, or as they transfer to magnetostatic space as a single quantity. The complete amount of the photon kinetic energy quantum could then continue remaining a single continuous quantity permanently linked through the central pointlike junction between the 3 spaces. This model of the double-particle photon can now be seen as displaying transverse wave behavior with a frequency related to the amount of energy that its quantum possesses, while at the same time displaying longitudinal particle behavior with longitudinal inertia related to the total amount of energy that its quantum possesses and transverse inertia related to half this amount, which conforms to all experimentally observed characteristics of the photon.

\section{Defining a Major Unit Vectors Superset}

The traditional $\hat{\mathbf{i}}, \hat{\mathbf{j}}$ and $\hat{\mathbf{k}}$ unit vectors previously mentioned, were of course defined to represent vectorial properties in normal 3D space. But in this expanded 3-spaces geometry, both new spaces also require their own internal minor unit vectors set.

So let's define a new superset of major unit vectors that will identify the three orthogonal spaces with capital letters as $\hat{\mathbf{I}}, \hat{\mathbf{J}}$ and $\hat{\mathbf{K}}$, so that each minor local $\hat{\mathbf{i}}, \hat{\mathbf{j}}$ and $\hat{\mathbf{k}}$ unit vectors set becomes subordinated to the major unit vector specific to its local space, all 12 resulting unit vectors ( 3 major and 9 minor) being of course drawn from the same origin $\mathrm{O}$ corresponding to the point-like junction between the 3 spaces (Figure 7).

Each of the three orthogonal minor unit vectors subsets (shown in the drawing as being half folded (let's remember the umbrella analogy), that is $\mathbf{I}-\mathbf{i}, \mathbf{I}-\mathbf{j}, \mathbf{I}-\mathbf{k}$, for normal space $\mathbf{J}-\mathbf{i}, \mathbf{J}-\mathbf{j}, \mathbf{J}-\mathbf{k}$ for electrostatic space and $\mathbf{K}-\mathbf{i}, \mathbf{K}-\mathbf{j}, \mathbf{K}-\mathbf{k}$ for magnetostatic space, allows defining the vectorial magnitude of the energy of a particle in any one of the three orthogonal coexisting spaces at any given moment.

This is how the vectorial relation drawn from Lorentz becomes in this expanded space geometry:

$$
\mathbf{E} \overrightarrow{\mathbf{J}} \times\left(\frac{-1}{\mathbf{B}}\right) \overrightarrow{\mathbf{K}}=\mathrm{v} \overrightarrow{\mathbf{I}}
$$

\section{Electromagnetic Oscillation Energy-driven rather than Fields-driven}

Now that we can view the photon kinetic energy quantum as a single continuous quantity permanently linked through the central point-like junction between the 3 spaces, comes into question the issue that the part of this amount oscillating between electrostatic and magnetostatic spaces must display distinct and apparently irreconcilable electric and magnetic properties that can continue being represented as reciprocally induced by the other aspect as in classical electromagnetism, that is, by apparent mutual "fields" interaction.

For example, if kinetic energy was a material incompressible in volume on top of its fundamental property of always tending to remain in motion, the local oscillation between both electrostatic and magnetostatic spaces of any quantity of this energy could possibly be

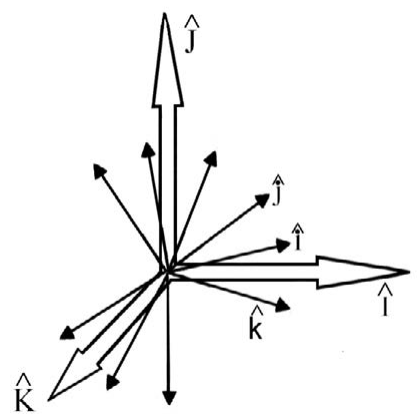

Figure 7: Major and minor unit vectors sets applicable to the 3-spaces model. 
forced uniquely by a property of this energy to always tend to remain in motion.So, instead of a relation of mutual orthogonal induction between two fundamentally different electric and magnetic "fields" as Maxwell's theory assumes, this relation would be one of cyclic translation of this energy between both orthogonal extra spaces (Figure 8).

That is, an energy that would always conserve the characteristics it originally possessed before it was quantized to become a photon, but that would give the impression of having alternately all of the electric set of characteristics when momentarily present in electrostatic space, and then, all of the magnetic set of characteristics when momentarily present in magnetostatic space; but whose high frequency cyclic translation between the two states (between the two spaces in reality) would create the impression at our macroscopic level of the simultaneous and permanent presence of both fields of Maxwell's theory inducing each other.

This would negate in no way the usefulness of fields representations. Fields would simply take second seat to the now self-forced motion of the kinetic energy proper becoming more fundamental, operating as a primary cause of the electromagnetic oscillation, being perceived momentarily as "electric energy" as it transits within electrostatic space and momentarily as "magnetic energy" as it transits within magnetostatic space.

It seems entirely conceivable that such a high frequency cyclic translation process of a discrete quantity of incompressible energy between these two spaces, could explain the frequency of the photon and all other observed phenomena while preserving the usefulness of the traditional perception of the so convenient and precise electric and magnetic fields that would mutually induce each other, but would also open up an entirely new range of possibilities, a few of which will be discussed later. Maxwell's four original equations would remain totally valid in this new perspective, since his second equation $(\nabla \times E=-\partial B / \partial t$ ) derived from Faraday's induction law, does not even mandate that both fields be in phase, since it directly accepts the opposite relation, that of reciprocal interaction of both fields when out of phase by $180^{\circ}$ as is being considered here.

\section{Underlying Kinetic Energy Circulation}

Let us now summarize the inner motion of kinetic energy within the structure of the double-particle photon.

This motion can be summarized as 4 distinct steps:

(a) The two half-photons having reached the farthest distance that they can reach within electrostatic space.

(b) The two half-photons closing in towards each other in

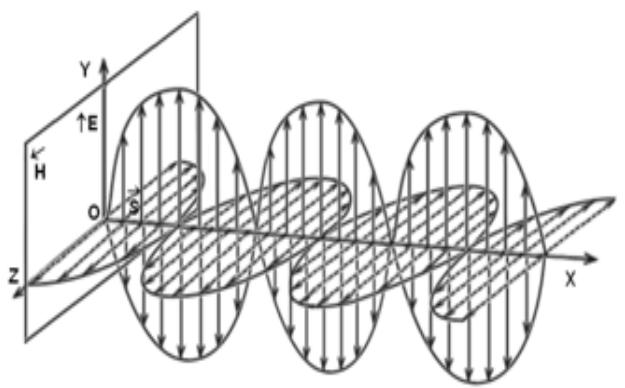

Figure 8: Electric and magnetic fields $180^{\circ}$ out of phase in the 3-spaces model.

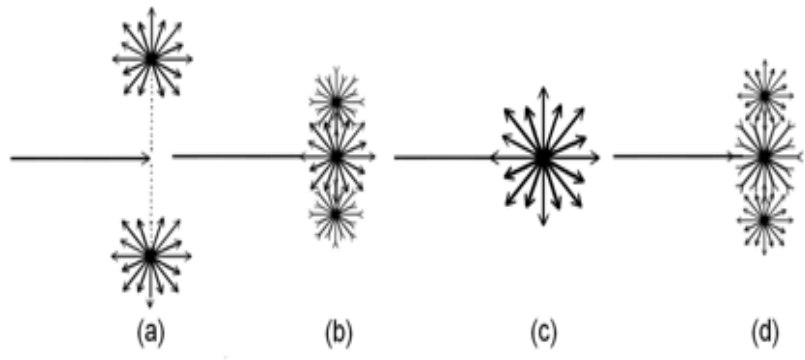

Figure 9: The complete cycle of kinetic energy circulation within the structure of the double-particle photon.

electrostatic space as their energy starts transferring omnidirectionally into magnetostatic space.

(c) The total complement of the two half-photons' energy having now completely crossed over into magnetostatic space, now making up the single spherical magnetic space component.

(d) The energy present in magnetostatic space starting to cross over back into electrostatic space as two separate half-photons.

(a) and (a) again as the cycle completes, poised to start the whole sequence again at the frequency mandated by the amount of kinetic energy making up the photon's energy quantum.

All through this process, the other half of the photon's energy, which is permanently located within normal space, remains in stable unidirectional motion, propelling the oscillating half at the speed of light in normal space vacuum.

\section{Applying Plane Wave Treatment to the Permanently Localized Double Particle Photon}

A point of particular interest with this internal photon structure is that it allows continued use of the plane wave analogy, but in which at any given instant of the cycle, the product of the electric and magnetic fields remains constant over the plane intersecting the central junction, perpendicularly to the direction of propagation of the photon (Figure 10). The energy of the photon would behave with respect to this plane as if it was stationary, as it actually is in the reference frame of the moving point-like junction, with the associated benefit that this plane, just like the point-like junction, can regardless continue moving at the speed of light in normal 3D space (along the $\mathrm{X}$ - $\mathrm{x}$ axis).Also, we can observe that the product of the projections on the transverse plane of the electric and magnetic oscillating energy will be constant and consequently will not fluctuate over time as is the case with classical in-phase wave front plane wave treatment.

In this model, the magnitude of the Poynting vector will thus be constant all through the electromagnetic cycle of any localized photon at the following value

$$
\mathbf{S}=\frac{\mathbf{E} \mathbf{B}}{2 \mu_{0}}
$$

instead of fluctuating over time as in classical electromagnetism, since one more characteristic of applying plane wave treatment to the double-particle photon in motion is that the value of $\mathbf{S}$ corresponds by structure very precisely to the average value of the intensity of the "wave" in classical electromagnetism [20].

Let us note here that this measured intensity is directly reconcilable with the conclusion of this model according to which only half of the 
energy of a photon would be oscillating to and fro between electrostatic and magnetostatic states while the other half would not be oscillating but would remain stable and moving unidirectionally to simply propel the oscillating half.

\section{The Double-particle Photon involves 2 Charges}

It is highly interesting to note that the new equation for free moving energy derived from Paul Marmet's work in a separate analysis ([10], equation (11)) involves by structure two interacting charges:

$$
E=h f=\frac{h c}{\lambda}=\frac{e^{2}}{2 \varepsilon_{0} \alpha \lambda}
$$

The very form $e^{2}$ reveal that both charges associated to a free moving electromagnetic energy quantum have to be identical, and can effectively be neutral $|\mathrm{e}|^{2}$ as hypothesized by de Broglie. This is what leads to the conclusion that it is possible that the opposite signs of a decoupling pair (positron + and electron -) could be acquired as the pair decouples, which is currently at odds with current axiomatic beliefs, but is in perfect harmony with de Broglie's conclusion that the double-particle photon charges should be neutral.

Let's also note that in this equation, fine structure constant $\alpha$ is related to the transverse amplitude of the electromagnetically oscillating half of the double-particle photon energy, an amplitude which in turn directly relates to the lower limit of spherical integration of the energy of a discrete localized electromagnetic particle ([10], Extended Abstract, and equations (1) to (11)).

\section{Defining The Double-particle Photon LC Equation and Local Fields Equation}

\section{Macroscopic LC circuits}

When an inductor coil is connected to a charged capacitor, it is well verified experimentally that the capacitor will completely discharge into the inductor coil as the current in the coil wire establishes a magnetic field in the surrounding space. When the potential difference between the capacitor terminals reaches zero, the magnetic field that just reached maximum about the inductor coil will now start decreasing, thus inducing a reverse current in the coil wire that will have completely recharged the capacitor when the magnetic field has completely disappeared, a behavior in complete agreement with $180^{\circ}$ out of phase electromagnetic cycling in this 3-spaces model (Figure 8).

The capacitor will now start discharging again into the inductor coil and the process would repeat indefinitely in theory if no energy was lost in the process, a loss that always occurs in lab experiments due to the resistance and eventual heating of the coil wire and radiating of this energy into surrounding space. It is well understood however that if no energy was lost due to the coil wire resistance, the total amount of energy in the system would remain stable and be permanently conserved, which would keep the cycle going forever.

\section{The Photon as a LC oscillator}

Let us now transpose this LC behavior to the double-particle photon. Contrary to the coil wire of a LC circuit made of a capacitor and an inductor coil, it can be assumed that the point-like junction between the three spaces of the expanded 3-spaces geometry will offer no resistance to the passage of the photon's oscillating energy, since it is well established that a photon's energy remains constant from emission to capture, however long the time elapsed since its emission and whatever distance it could have traveled, barring losses due to red shifting, gains due to blue shifting and losses due to gravity induced changes in direction.

The classical equation representing the maximum energy stored in the capacitor of a LC circuit at the beginning of the cycle is:

$$
E_{\mathbf{E}(\max )}=\frac{q^{2}}{2 C}
$$

and the equation representing the maximum energy stored in the magnetic field of the coil when the capacitor has been emptied of its charge is:

$$
E_{\mathbf{B}(\max )}=\frac{L i^{2}}{2}
$$

In the context of LC behavior applied to a localized photon's energy, where no energy can be lost through heating of a non-existent coil wire and considering that both quantities represent the same half quantum of the photon's energy oscillating between these two maxima, we can then equate:

$$
E_{\mathrm{E}(\max )}=E_{\mathrm{B}(\max )}=E_{\mathrm{E}}+E_{\mathrm{B}}=E_{\mathrm{EB}}
$$

\section{Defining the photon capacitance $(C)$}

As established in a separate analysis [12], only half of a photon's energy cyclically oscillates between electric and magnetic states. So making use of the free energy equation previously mentioned derived from Marmet's work ([10], equation (11)), that is:

$$
E=\frac{e^{2}}{2 \varepsilon_{0} \alpha \lambda}
$$

that we will divide by 2 , to represent only the oscillating half of the photon's energy, and equate to equation (18) for capacitance, which represents the same half the photon's energy, that is, the two charges of the photon at their maximum value, we obtain:

$$
E_{\mathbf{E B}}=\frac{E}{2}=\frac{q^{2}}{2 C}=\frac{e^{2}}{4 \varepsilon_{0} \alpha \lambda}
$$

We can then isolate:

$2 C=4 \varepsilon_{0} \alpha \lambda$

and finally obtain:

$C=2 \varepsilon_{0} \alpha \lambda$ Farad 
which allows calculating the capacitance of any localized photon from its wavelength and the permittivity constant of vacuum $\left(\varepsilon_{0}\right)$.

\section{Defining the photon inductance $(\mathrm{L})$}

Since the angular frequency of a LC oscillator is obtained from the following equation:

$$
\omega=\sqrt{\frac{1}{L C}}
$$

we can separately calculate the angular frequency of a localized photon's energy from $\omega=2 \pi f / \alpha$, or better yet, in context, from $\omega=2 \pi c / \alpha \lambda$ (since we must use here the $\alpha$ related transverse amplitude (See Section 16) of the cycling frequency calculated from the wavelength of a localized photon's energy which is $f=c / \lambda)$. So we can write:

$$
\omega=\frac{2 \pi c}{\dot{a} \lambda}=\sqrt{\frac{1}{L C}}
$$

By squaring this last equation and replacing $C$ by its value defined in equation (24) as $2 \varepsilon_{\alpha} \alpha \lambda$, we can isolate $L$ in equation (26) and define the following equation:

$$
L=\frac{\alpha^{2} \lambda^{2}}{C 4 \pi^{2} c^{2}}=\frac{\alpha \lambda}{\varepsilon_{0} 8 \pi^{2} c^{2}}
$$

Knowing that $\varepsilon_{o} c^{2}=1 / \mu_{o}$ and substituting this value into equation (27) to introduce permeability constant $\mu_{\mathrm{o}}$, we finally obtain:

$$
L=\frac{\mu_{0} \alpha \lambda}{8 \pi^{2}} \quad \text { Henry }
$$

which allows calculating the inductance of any localized photon from its wavelength and the permeability constant of vacuum $\left(\mu_{0}\right)$

\section{The Photon maximum displacement current ( $i)$}

Having established how to calculate inductance $L$ for a localized photon, we can now determine the maximum current $i$ involved from the equation giving the maximum energy momentarily stored in the magnetic field. So, from equation (19):

$$
E_{\mathbf{B}(\max )}=\frac{L i^{2}}{2}
$$

we can isolate $i$, and knowing that $E_{\mathbf{B}(\max )}=E_{\mathrm{EB}}$ from equation (20), the value of $L$ from equation (28), and knowing also that $\varepsilon_{0} \mu_{0}=1 / c^{2}$, we can derive the localized photon's maximum displacement current:

$$
\begin{aligned}
& i=\sqrt{\frac{2 E_{E B}}{L}}=\sqrt{2 \frac{e^{2}}{4 \varepsilon_{0} \lambda \alpha} \frac{8 \pi^{2}}{\mu_{0} \lambda \alpha}} \\
& =\sqrt{\frac{4 \pi^{2} e^{2}}{\varepsilon_{0} \mu_{0} \alpha^{2} \lambda^{2}}}=\sqrt{\frac{4 \pi^{2} e^{2} c^{2}}{\alpha^{2} \lambda^{2}}}=\frac{2 \pi e c}{\alpha \lambda} \text { Ampere }
\end{aligned}
$$

\section{The photon general LC equation}

Remembering that the of $E_{\mathrm{E}}$ and $E_{\mathrm{B}}$ is permanently constant as established with equation (20), we can now write:

$$
\begin{aligned}
E_{\mathbf{E B}} & =E_{\mathbf{E}}+E_{\mathbf{B}} \\
& =\left[2\left(\frac{e^{2}}{4 C}\right)_{\mathrm{Y}} \cos ^{2}(\omega t)+\left(\frac{L i^{2}}{2}\right)_{\mathrm{Z}} \sin ^{2}(\omega t)\right]
\end{aligned}
$$

Where $t$ is the time for one cycle, corresponding to $1 / f$, or when defined as a function of $\lambda$ as required here, corresponding to $t=\lambda / c$, and where the electric aspect needs by structure to be split into two equal quantities moving in opposite directions within electrostatic space (Y-space).

Since this energy corresponds to only half of the energy of the photon, we must finally add the other half, which is unidirectional and permanently localized within normal space (X-space) to obtain the total energy of the photon. Let's now also introduce the required set of directed unit vectors to completely represent the various directions of motion of the energy within the 3 -spaces structure:

$$
E \vec{I} \vec{i}=\left(\frac{h c}{2 \lambda}\right)_{\mathrm{X}} \vec{I} \vec{i}+\left[\begin{array}{l}
2\left(\frac{e^{2}}{4 C}\right)_{\mathrm{Y}}(\vec{J} \vec{j}, \vec{J} \stackrel{\leftarrow}{j}) \cos ^{2}(\omega t) \\
+\left(\frac{L i^{2}}{2}\right)_{\mathrm{Z}} \stackrel{\leftrightarrow}{K} \sin ^{2}(\omega t)
\end{array}\right]
$$

Equation (32) is the most detailed and general equation, all terms of which being function of a single variable, that is, the photon wavelength $\lambda$, that can be established for the internally cycling energy of the permanently localized double-particle photon of de Broglie's hypothesis in this expanded space geometry, and where indices $\mathrm{X}, \mathrm{Y}$ and $Z$ represent the three mutually orthogonal spaces into which the kinetic energy quantum is in standing motion. All that is required now to observe how the energy oscillates between electric and magnetic states is to cyclically vary $\mathrm{t}$ from 0 to $\lambda / c$. This equation allows clearly understanding why the Poynting vector becomes totally stable when de Broglie's hypothesis is taken into account, at a value equal to the average value of this vector in classical Maxwell. This stability is due to the fact that at any given moment, the sum of capacitance and inductance energies is always exactly equal to half a photon's energy.

\section{The photon general local fields equation}

Equation (32), making use of the less familiar energy inductance and capacitance that were required to describe the double-particle photon kinetic energy electromagnetic oscillation, would gain in handiness if converted to use the more familiar electric (E) and magnetic (B) fields expressions for energy.

For a photon moving in straight line, it is well established that both electric and magnetic aspects of its internal energy have to be of equal density as described in ([10], equation (35)):

$$
\mathbf{u}_{\mathbf{B}}=\mathbf{u}_{\mathbf{E}}=\frac{\mathbf{B}^{2}}{2 \mu_{0}}=\frac{\varepsilon_{0} \mathbf{E}^{2}}{2}
$$

Given that an energy density is an energy value divided by a volume, the fields related expressions for a photon's energy can be recovered by multiplying these density expressions by the related theoretical stationary isotropic volume that this incompressible oscillating kinetic energy quantum would occupy if it was immobilized as a sphere of isotropic density ([10], equation (40h)):

$$
V=\frac{\alpha^{5}}{2 \pi^{2}}
$$

which, when multiplying the $\mathbf{u}_{\mathrm{B}}$ and $\mathbf{u}_{\mathrm{E}}$ fields energy density values expressed in equation (33) by this volume, will provide the required fields related energy values:

$$
E_{\mathbf{E}}=\frac{\varepsilon_{0} \mathbf{E}^{2}}{2} V \quad \text { and } \quad E_{\mathbf{B}}=\frac{\mathbf{B}^{2}}{2 \mu_{0}} V
$$

This in turn allows the following conversion of equation (32) to a more familiar fields expression: 


$$
E \vec{I} \vec{i}=\left(\frac{h c}{2 \lambda}\right)_{\mathrm{X}} \vec{I} \vec{i}+\left[\begin{array}{l}
2\left(\frac{\varepsilon_{0} \mathbf{E}^{2}}{4}\right)_{\mathrm{Y}}(\vec{J} \vec{j}, \vec{J} \stackrel{\leftarrow}{j}) \cos ^{2}(\omega t) \\
+\left(\frac{\mathbf{B}^{2}}{2 \mu_{0}}\right)_{\mathrm{Z}} \stackrel{\leftrightarrow}{K} \sin ^{2}(\omega t)
\end{array}\right] V
$$

where the photon electric field is expressed as:

$$
\mathbf{E}=\frac{\pi e}{\varepsilon_{0} \alpha^{3} \lambda^{2}} \text { from }([10], \text { equation }(40))
$$

and the photon magnetic field is expressed as:

$$
\mathbf{B}=\frac{\mu_{0} \pi e c}{\alpha^{3} \lambda^{2}} \text { from }([10], \text { equation (34)) }
$$

\section{The photon default self-guiding in straight line and self- propelling at the speed of light}

It is quite interesting to observe that the default equal density by structure of both electric and magnetic fields of the double-particle photon directly explains why photons self-guide in straight lines when no outside force is acting on them, in conformity with Maxwell's fourth equation. The manner in which the trajectories of elementary electromagnetic particles can be very precisely programmed, by causing the default equal densities of both ambient electric and magnetic fields to vary from their equilibrium state, is completely described in any good textbook on high energy accelerators, such as the wonderfully made "Principles of Charged Particle Acceleration" by Stanley Humphries [21]. The mechanics of the natural variation of this default equilibrium of the density of both fields in the 3-spaces model for electromagnetic particles subjected to transverse interaction is described in a separate paper [22]. In addition to providing the previously described constant magnitude for the Poynting vector, it is also interesting to observe that this internal structure also provides a mechanical explanation to the stability of the speed of light of free moving electromagnetic energy in vacuum. As mentioned previously, a separate analysis [12] mathematically demonstrates why the speed of light of localized photons can be explained only if its kinetic energy is distributed as one half unidirectionally moving in space, propelling an equal amount of energy captive in transverse electromagnetic oscillation. It can be hypothesized that the 3-spaces structure itself acts as a set of communicating vessels through the common central junction, which would be offering zero resistance to the passage of energy, since objective reality shows that no energy is lost during free moving energy electromagnetic oscillation, and that this junction always allows the energy of the photon to remain in some form of permanent equilibrium between the 3 spaces, an equilibrium that would constantly seek to keep the photon's energy split into two equal amounts between $\mathrm{X}$-space and YZ-spaces, even during energy losses or gains events related to red and blue shifting due to gravitational interaction. When energy is lost by a photon as witnessed by a displacement towards the red of its frequency or gained as witnessed by a displacement towards the blue of its frequency, the half-half $\mathrm{X}$ vs $\mathrm{YZ}$ equilibrium would be maintained by the required amount of kinetic energy seeping through the $\mathrm{X}-\mathrm{YZ}$ junction in the direction required to constantly restore this equilibrium. This would directly explain why all photons self-propel, so to speak, at the same constant "equilibrium" velocity, which is of course the speed of light.

Now this brings up the old issue of what this "equilibrium" constant velocity of photons in vacuum (free moving kinetic energy) is relative to in reality. Is it relative to the medium? To the point of emission? To the point of absorption? To the observer? To this or that reference frame, or multiple reference frames, inertial, non inertial, Galilean, moving or not, etc. A deeply ingrained habit has developed since the beginning of the 20th century to hypothesize various reference frames in attempts to make sense of the experimentally observed data. But in physical reality, velocity depends on only one criterion: the actual presence of translational kinetic energy. If translational kinetic energy is present and if the local electromagnetic equilibrium allows it, there will be velocity in vacuum, relative to there being absence of translational kinetic energy, irrespective of any hypothesized reference frame or frames.

The absolute lower velocity limit, as seen from this perspective, would be an electron possessing zero translational kinetic energy in excess the energy making up its rest mass. Of course, such an electron totally deprived of translational kinetic energy can only be theoretical, because all massive particles are subject to gravitational or electrostatic acceleration in physical reality from the moment they start existing.

The absolute upper velocity limit involving electromagnetic oscillation is reached when an amount of translational (aka unidirectional) kinetic energy propels an equal amount of kinetic energy captive in transverse electromagnetic oscillation, that is, a free moving photon for example, as described in this paper.

The only other possible case between these two limits involving electromagnetic oscillation, applies to an amount of kinetic energy captive in transverse electromagnetic oscillation being propelled by a lesser amount of translational kinetic energy, such as the kinetic energy making up the rest mass of an electron, plus the transversely oscillating half of its carrier-photon's kinetic energy, both quantities being propelled by the unidirectional half of the carrier-photon's quantum of kinetic energy. The velocity of such a system will mandatorily lie between zero and asymptotically close to the speed of light, a process whose mechanics is described in a separate paper [12].

Finally, there remains one case of kinetic energy whose motion seems not to involve any electromagnetic oscillation and for which there consequently also seems not be any limiting factor on the velocity. It is the case of escaping neutrino energy, whose mechanics of liberation in the 3-spaces model is described in a separate paper [23].

\section{The deflection angle of photons' trajectories}

All of these considerations bring us to re-examine the case of light deflection experimentally verified for the first time in 1919 by Eddington and many others afterwards, during solar eclipses [24], to confirm a prediction of Einstein to the effect that light from far stars can be deflected by gravity and that this deflection could be measured, for example, as light closely grazes the mass of the Sun.

According to Newton's theory, the inertia of all bodies is deemed to be omnidirectional so a body should resist any change in its state of motion with the same intensity to a force acting on it, whichever direction it is being applied from. Associating mass to photons for the purpose of calculation, Einstein then applied the same logic to their total energy, assuming that the total complement of a photon's energy is subject to transverse interaction when flying by a celestial body as a function of the inverse square of the distance between them. His calculation then gave an estimated deflection angle of 0 " 83 arc second as as mentioned in a paper [25] that he published in 1911, that is, an angle twice shallower than the actual angle that will be observed in reality, which seemed to invalidate Newton's mechanics at the fundamental 
particles' level. Of course, he afterwards provided a different calculation, which gave an estimated double deflection angle of 1"75 arc second which is closer to reality, the supplementary deflection angle increment being considered an effect of the space-time curvature of his General Relativity Theory and a proof of the soundness of the theory.

Interestingly, as demonstrated in a separate analysis [12], the unidirectional half of the energy of the double-particle photon that must remain within normal $\mathrm{X}$-space by structure, is impervious to transverse interaction as demonstrated by Walter Kaufmann at the beginning of the $20^{\text {th }}$ century, as he carried out experiments by inducing varying amounts of kinetic energy into electrons [19]. When the trajectories of the moving electrons were not deflected (observations made by means of a bubble chamber), he found of course that the total longitudinal inertia of the particle involved the energy making up the rest mass of the electron plus the total amount of the added kinetic energy provided to the particle. But when the trajectories were deflected with sufficiently high velocities, he discovered that the transverse inertia of the particle involved less energy than this sum, which gave rise to the debate regarding "longitudinal mass" and "transverse mass", which led to the conclusion that mass was electromagnetic in nature. Close analysis shows that only part of the additional kinetic energy provided was involved in the transverse inertia component. Moreover, further analysis shows more precisely that at any velocity, exactly half of the additional kinetic energy provided converts to a momentary velocity related "relativistic" mass increment, which means that the other half of the additional kinetic energy, that is, the translational half of the kinetic energy provided, is totally impervious to transverse interaction while propelling the total amount of energy captive in the rest mass of the particle plus the momentary velocity related relativistic mass increment.

This means that the instantaneous relativistic mass of a moving particle can be directly measured only by means of transverse interaction since longitudinal inertia does not allow distinguishing the rest mass of the particle from the contribution from its carrying energy. This gave rise to the development of a new set of relativistic equations derived from electromagnetism which is complementary to that stemming from the Special Relativity Theory [12].

This new set can be summarized as follows in a form easy to manipulate with any scientific hand calculator. The full range of relativistic velocities can be obtained from this equation:

$$
f(x)=c \frac{\sqrt{4 a x+x^{2}}}{2 a+x}
$$

Where $f(x)$ is the relativistic velocity, "a" is the energy in joules contained in the rest mass of the electron (8.18710414E-14 joules) and " $x$ " is the kinetic energy provided in joules. " $c$ " is of course the speed of light in meters per second.

From equation (39) can be derived the following equation that allows calculating the kinetic energy that must be communicated to an electron for it to move at relativistic velocity $\mathrm{v}$, when only this velocity is known:

$$
\mathrm{x}=2 \mathrm{a}(\gamma-1)
$$

Where " $x$ " is the added kinetic energy, " $a$ " is the energy making up the rest mass of the electron and $\gamma$ is the Lorentz gamma factor. Any relativistic velocity plugged into the gamma factor will allow obtaining the amount of kinetic energy required for the particle to move at this velocity.
Let us note that the gamma factor would be much easier to deal with in equations if it was simplified to the following form which leaves only one fraction in the expression:

$$
\gamma=\frac{1}{\sqrt{1-\mathrm{v}^{2} / \mathrm{c}^{2}}}=\sqrt{\frac{\mathrm{c}^{2}}{\mathrm{c}^{2}-\mathrm{v}^{2}}}=\frac{\mathrm{c}}{\sqrt{\mathrm{c}^{2}-\mathrm{v}^{2}}}
$$

With the velocity related amount of kinetic energy calculated with equation (40), the following equation allows calculating the instantaneous relativistic mass of the particle for this relativistic velocity:

$$
\mathrm{m}_{\text {(rel) }}=\mathrm{m}_{0}+\frac{\mathrm{x}}{2 \mathrm{c}^{2}}
$$

The full range of relativistic velocities can also be obtained from the following equation by using the wavelengths of the energies involved:

$$
f(x)=c \frac{\sqrt{4 a x+a^{2}}}{2 x+a}
$$

where $f(x)$ is the relativistic velocity, "a" is the electron Compton wavelength (2.426310215E-12 m) and " $\mathrm{x}$ " is the wavelength of the total amount of kinetic energy provided to the particle. Finally, similarly to equation (40) being derived from equation (39), the following equation derived from equation (43) allows calculating the wavelength of the energy that must be communicated to an electron for it to move at relativistic velocity $\mathrm{v}$, when only this velocity is known:

$$
\lambda=\frac{\lambda_{C}}{2(\gamma-1)}
$$

Where " $\lambda$ " is the wavelength of the communicated energy, " $\lambda_{C}$ " is the electron Compton wavelength and " $\gamma$ " is the Lorentz factor. In relation with the analysis carried out in a separate paper [12], when the energy making up the rest mass of the electron is set to zero in equation (39), or rather, in its electromagnetic version ([12], equation (33)), we observe that the only velocity that can be obtained is $c$, that is, the speed of light. This means that the remaining transversaly measurable mass increment plus the transversaly undetectable but equal translational other half of the added energy involved behave like a free moving photon, displaying a longitudinal inertia corresponding to the total amount of energy involved, but a transverse inertia corresponding to only half of the total amount of energy involved. This observation with regards to the electron's carrying energy comes in support of the analysis carried out in this paper to the effect that free moving electromagnetic photons would, by similarity, have the same internal electromagnetic structure as massive particles' carrying energy. Consequently, if Einstein's calculations had been done with the "mass" of only the electromagnetically oscillating half of the photon's energy with regards to photons' trajectories deflection by celestial bodies, that is, the only part of photons' energy that seems to be sensitive to transverse interaction, then the 1"75 arc second deflection angle for photons flying closely by the Sun could have been directly obtained from classical mechanics without any need to resort to the General Relativity space-time curvature.

\section{Conclusion}

This paper is meant to show that it is possible to represent the permanently localized photon of de Broglie's hypothesis in a manner totally conform to Maxwell's equations. This particular solution requires expanding the local space geometry in a manner that allows the photon's kinetic energy quantum to behave in accordance with Maxwell's theory without changing in nature while at the same time 
displaying the mutually exclusive properties of electric and magnetic fields. Since energy can be represented in a number of ways as exemplified by the two currently available approaches mentioned in the introduction Section, solutions other than the one proposed here are of course possible. But hopefully, the non-exhaustive benefits mentioned in this paper that this new solution provides may help rekindle causality based fundamental research in the community, all the more so since this space geometry also allows a simple and logical mechanical explanation to the process of conversion of massless photons of energy $1.022 \mathrm{MeV}$ or more to pairs of massive electronpositron [13] that also structurally possess the dual wave-particle characteristics that characterize this model of the photon, and much more.

\section{References}

1. Einstein A (1953) Louis de Broglie, physicien et penseur. Éditions Albin Michel, Paris.

2. De Broglie $L$ (1993) La physique nouvelle et les quanta $\left(2^{\text {nd }} e d n\right)$. Flammarion France.

3. Buras AJ (2005) Photon Structure Functions: 1978 and 2005. Acta Physica Polonica B 37: 609 .

4. Sears F, Zemansky M, Young H (1984) University Physics (6 $6^{\text {th }}$ edn.). Addison Wesley, USA.

5. Lochak G (1992) Louis de Broglie, Flammarion, France.

6. Feynman R (1949) Space-Time Approach to Quantum Electrodynamics. Phys Rev lett 76: 769

7. Breidenbach M (1969) Observed Behavior of Highly Inelastic Electron-Proton Scattering. Phys Rev Lett 23: 935-939.

8. Greiner W, Reinhardt J (1994) Quantum Electrodynamics. Springer-Verlag.

9. Burke DL (1997) Positron Production in Multiphoton Light-by-Light Scattering. Phys Rev Let 79: 1626.

10. Michaud A (2007) Field Equations for Localized Individual Photons and Relativistic Field Equations for Localized Moving Massive Particles. International IFNA-ANS Journal 13: 23-140.
11. Marmet P (2003) Fundamental Nature of Relativistic Mass and Magnetic Fields. International IFNA-ANS Journal 9: 1-10.

12. Michaud A (2013) From Classical to Relativistic Mechanics via Maxwell. International Journal of Engineering Research and Development 6: 1-10.

13. Michaud A (2013) The Mechanics of Electron-Positron Pair Creation in the 3-Spaces Model. International Journal of Engineering Research and Development 6: 1-10.

14. Hanson G (1975) Evidence for Jet Structure in Hadron Production by e+ eAnnihilation. Phys Rev Let 35: 1609-1612.

15. Michaud A (2013) The Mechanics of Neutron and Proton Creation in the 3-Spaces Model. International Journal of Engineering Research and Development 7: 29-53.

16. Kotler S, Akerman N, Navon N, Glickman Y, Ozeri R (2014) Measurement of the magnetic interaction between two bound electrons of two separate ions. Nature magazine 510: 376-380

17. Michaud A (2013) On The Magnetostatic Inverse Cube Law and Magnetic Monopoles. International Journal of Engineering Research and Development 7: $50-66$.

18. Michaud A (2000) On an Expanded Maxwellian Geometry of Space. Fundamental Problems of Natural Sciences and Engineering, Russia.

19. Kaufmann W (1903) Über die "Elektromagnetische Masse" der Elektronen, Kgl. Gesellschaft der Wissenschaften Nachrichten. Mathem-Phys Klasse 2: 91-103.

20. Resnick R, Halliday D (1967) Physics. John Wyley and Sons, New York.

21. Humphries S (1986) Principles of Charged Particle Acceleration. John Wiley and Sons, New York

22. Michaud A (2013) On the Electron Magnetic Moment Anomaly. International Journal of Engineering Research and Development 7: 21-25.

23. Michaud A (2013) The Mechanics of Neutrinos Creation in the 3-Spaces Model. International Journal of Engineering Research and Development 7: 1-8.

24. Ohanian HC, Ruffini R (1994) Gravitation and Spacetime ( $2^{\text {nd }}$ edn.). Norton New York.

25. Einstein A (1911) About the influence of gravity on the propagation of light Annals of Physics 340: 898-908. 